\title{
Association of Inflammation With Pronociceptive Brain Connections in Rheumatoid Arthritis Patients With Concomitant Fibromyalgia
}

\author{
Chelsea M. Kaplan, ${ }^{1}$ iD Andrew Schrepf, ${ }^{1}$ Eric Ichesco, ${ }^{1}$ Tony Larkin, ${ }^{1}$ Steven E. Harte, ${ }^{1}$ Richard E. Harris, ${ }^{1}$ \\ Alison D. Murray, ${ }^{2}$ Gordon D. Waiter, ${ }^{2}$ Daniel J. Clauw, ${ }^{1}$ and Neil Basu ${ }^{3}$
}

Objective. Rheumatoid arthritis (RA) patients with concomitant fibromyalgia (FM) exhibit alterations in brain connectivity synonymous with central sensitization. This study was undertaken to investigate how peripheral inflammation, the principal nociceptive stimulus in RA, interacts with brain connectivity in RA patients with FM.

Methods. RA patients with concomitant FM and those without FM (FM+ and FM-, respectively; $\mathrm{n}=27$ per group) underwent functional connectivity magnetic resonance imaging. Seed-to-whole-brain functional connectivity analyses were conducted using seeds from the left mid/posterior insula and left inferior parietal lobule (IPL), which are regions that have been previously linked to FM symptoms and inflammation, respectively. The association between functional connectivity and erythrocyte sedimentation rate (ESR) was assessed in each group separately, followed by post hoc analyses to test for interaction effects. Cluster-level, family-wise error (FWE) rates were considered significant if the $P$ value was less than 0.05 .

Results. The group of RA patients with FM and those without FM did not differ by age, sex, or ESR $(P>0.2)$. In FM+ RA patients, increased functional connectivity of the insula-left IPL, left IPL-dorsal anterior cingulate, and left IPL-medial prefrontal cortex regions correlated with higher levels of ESR (all FWE-corrected $P<0.05$ ). Post hoc interaction analyses largely confirmed the relationship between ESR and connectivity changes as FM scores increased.

Conclusion. We report the first neurobiologic evidence that FM in RA may be linked to peripheral inflammation via pronociceptive patterns of brain connectivity. In patients with such "bottom-up" pain centralization, concomitant symptoms may partially respond to antiinflammatory treatments.

\section{INTRODUCTION}

Rheumatoid arthritis (RA) is known for its heterogeneous clinical presentation, with some patients reporting pain localized to affected joints and others reporting widespread hyperalgesia, fatigue, and cognitive difficulties. This latter phenotype has remarkable similarities to symptoms observed in fibromyalgia (FM), and indeed the reported prevalence of concomitant FM in

\footnotetext{
Supported by Pfizer.

${ }^{1}$ Chelsea M. Kaplan, PhD, Andrew Schrepf, PhD, Eric Ichesco, BS, Tony Larkin, BS, Steven E. Harte, PhD, Richard E. Harris, PhD, Daniel J. Clauw, MD: University of Michigan, Ann Arbor; ${ }^{2}$ Alison D. Murray, MD, PhD, Gordon D. Waiter, PhD: University of Aberdeen, Aberdeen, UK; ${ }^{3}$ Neil Basu, MD, PhD: University of Glasgow, Glasgow, UK.

Drs. Kaplan and Schrepf contributed equally to this work.

Dr. Harte has received consulting fees from Pfizer, Analgesic Solutions, Aptinyx, and deCode Genetics (less than $\$ 10,000$ each) and research support from Aptinyx, Cerephex, Eli Lilly, Forest Laboratories, and Merck. Dr. Harris has received consulting fees (less than $\$ 10,000$ ) and research support from Pfizer. Drs. Murray, Waiter, and Basu have received research support from
}

RA patients ranges from $12 \%$ to $48 \%$, compared to $2-8 \%$ in the general population (1). Moreover, we recently showed that one of the chief neurobiologic signatures of FM (increased default mode network [DMN] to insula functional connectivity) also occurs in RA patients in proportion to their FM symptoms (2).

In the context of RA, it has long been hypothesized that peripheral inflammatory nociceptive processes sensitize the central nervous system (CNS) via pronociceptive CNS pathways and drive

Pfizer. Dr. Clauw has received consulting fees from and/or served as an expert witness for Tonix, Aptinyx, Daiichi Sankyo, Samumed, Intec Pharma, Eli Lilly, Zynerba, Williams \& Connolly LLP, and Therevance (less than $\$ 10,000$ each) as well as Pfizer and Nix Patterson LLP (more than $\$ 10,000$ each) and has also received research support from Pfizer and Aptinyx. No other disclosures relevant to this article were reported.

Address correspondence to Chelsea M. Kaplan, PhD, University of Michigan, Chronic Pain and Fatigue Research Center, Department of Anesthesiology, Lobby M, PO Box 385, 24 Frank Lloyd Wright Drive, Ann Arbor, MI 48106. E-mail: chelsmar@umich.edu.

Submitted for publication November 27, 2018; accepted in revised form August 1, 2019. 
the occurrence of concomitant FM (3). This suggests that individual differences in how the brain responds to inflammation may be critical to understanding concomitant FM in RA.

We recently provided some of the first evidence that functional brain connections are substantially altered in RA patients with high levels of peripheral inflammation (4). In the present study, we expanded on that effort by exploring the effect of the interaction between functional brain connectivity and inflammation on FM symptoms in the same cohort of RA patients. These analyses were designed to identify patterns of brain connectivity in RA that promote core symptoms of $\mathrm{FM}$ in response to inflammation. We hypothesized that higher inflammation would be associated with distinct changes in connectivity from the insula (a pronociceptive brain region) and left inferior parietal lobule (IPL; a region associated with the neural response to peripheral inflammation in RA patients) to other brain regions in patients with a high level of FM symptoms.

\section{PATIENTS AND METHODS}

Patients. RA patients were approached through a UK regional rheumatology service. Patients were considered eligible if they met the American College of Rheumatology (ACR)/ European League Against Rheumatism 2010 classification criteria for RA (5). This study was designed to examine the neural correlates of fatigue in RA; therefore, patients had to have had a clinically significant level of fatigue for at least 3 months (defined as a score of $>3$ on the Chalder Fatigue Binary Scale [6]). The analyses presented in this report address alternate hypotheses regarding inflammation and centralized pain in RA. Exclusion criteria were contraindications to magnetic resonance imaging (MRI), left-handedness, or the presence of an alternative medical explanation for fatigue. A total of 73 RA patients fulfilled these criteria, and 54 patients ( 41 female) completed the entire study. Ethical approval for the study was obtained from the North of Scotland Research Ethics Committee. All participants provided written informed consent in accordance with the Declaration of Helsinki.

Clinical evaluation. All consenting patients underwent a clinical evaluation and a functional MRI (fMRI) brain scan. FM diagnosis was assessed using the ACR modified 2010 criteria (7), which combine a measure of widespread pain (number of painful sites [range 0-19]) with a symptom severity scale (e.g., fatigue, subjective cognitive problems, headache, poor mood; scores range from 0 to 12). A combined score of $\geq 13$ was used as the diagnostic cutoff for FM (7). Twenty-seven RA patients met the criteria for FM (FM+), and 27 did not (FM-). Inflammation was measured using the erythrocyte sedimentation rate (ESR), a measure of systemic inflammation that is commonly used to diagnose and monitor chronic inflammatory diseases such as RA. A trained phlebotomist collected venous blood samples during standard clinic hours, and blood samples were immediately processed and analyzed for the calculation of ESR (via the Westergren method).

\section{Functional MRI data acquisition and preprocessing.} Functional MRI data were collected using an Achieva 3.0T X-series MR system (Philips Medical) with an 8-channel phased-array head coil. Each patient underwent an 11-minute functional scan while performing the Paced Auditory Serial Attention Test (PASAT), a validated measure of cognitive function. The PASAT was administered in a block design with three 3-minute "on" periods, interspersed with four 30-second rest or "off" periods. During the "on" periods, a series of numbers were audibly presented, and patients were asked to sum consecutive numbers and to press a button every time 2 consecutive numbers summed to 10. Concurrently, patients were shown a distracting visual stimulus of random, rapidly changing numbers that were intended to increase task difficulty. The functional images were acquired using a 3-dimensional (3-D) T2*-weighted gradient-echo single-shot echo-planar imaging pulse sequence with the following parameters: repetition time (TR) 3,000 msec, echo time (TE) $30 \mathrm{msec}$, flip angle $90^{\circ}$, in-plane

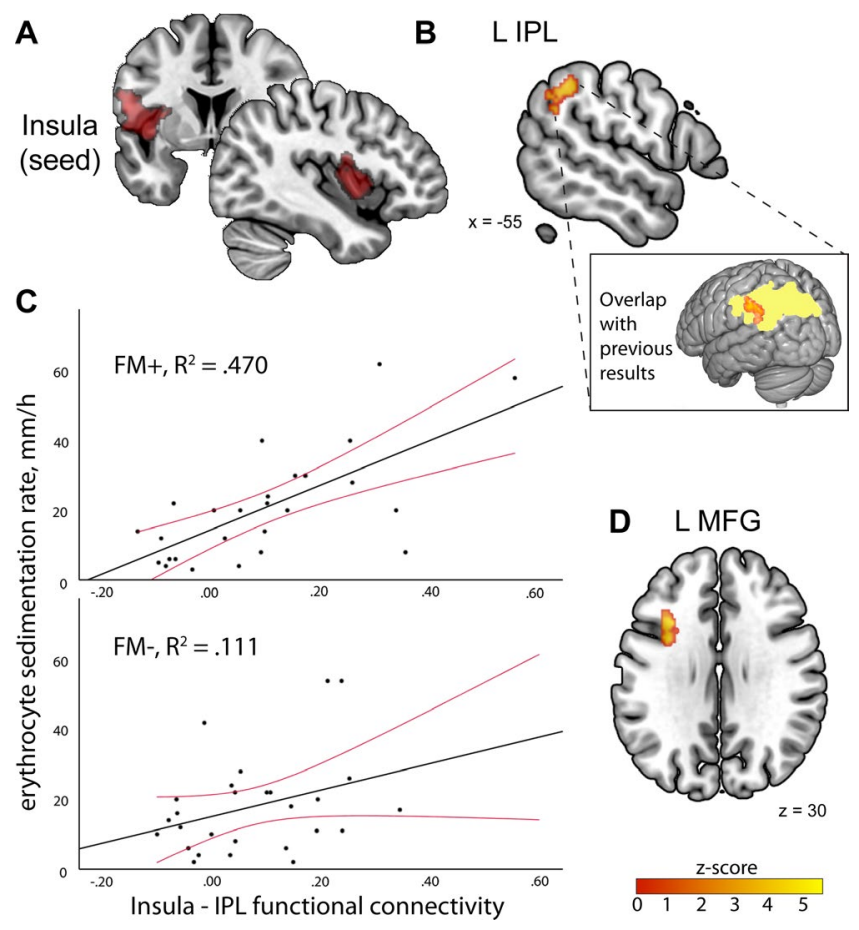

Figure 1. A and $\mathbf{B}$, Correlation of higher levels of peripheral inflammation with increased brain connectivity between the left mid/ posterior insula $(\mathbf{A})$ and the left inferior parietal lobule $(L I P L)(B)$ in rheumatoid arthritis (RA) patients with concomitant fibromyalgia (FM). Inset in B shows overlap of the left IPL cluster with previous results (4), demonstrating that numerous brain networks were hyperconnected to the same region of the IPL in RA patients with high levels of inflammation. C, Scatterplots showing correlation between erythrocyte sedimentation rate and insula-IPL connectivity in RA patients with or without FM; 95\% confidence intervals are displayed in red. D, Significant positive relationship between insula-left middle frontal gyrus (MFG) connectivity and peripheral inflammation in RA patients with FM. 
Sensitivity Encoding (SENSE) acceleration 2, field of view (FOV) $240 \mathrm{~mm}$, matrix size $128 \times 128$ pixels with 30 slices, voxel size $1.88 \times 1.88 \times 5 \mathrm{~mm}^{3}$, and 226 volumes. A high-resolution structural T1-weighted fast field-echo 3-D structural scan was collected for normalization (TR $8.2 \mathrm{msec}$, TE $3.8 \mathrm{msec}$, inversion recovery time 1,018 msec, flip angle $8^{\circ}$, FOV $240 \mathrm{~mm}$, matrix size $240 \times$ 240 pixels with 160 slices, and voxel size $0.94 \times 0.94 \times 1 \mathrm{~mm}^{3}$ ).

Functional MRI data were preprocessed using Statistical Parametric Mapping (SPM version 8; Wellcome Department of Cognitive Neurology, London, UK) running on MatLab R2014a (MathWorks), as previously described $(2,4)$. Briefly, the first 4 volumes were discarded to avoid equilibration effects, and the remaining 222 functional images were realigned to the first image. The structural image was coregistered to a mean functional image and then segmented. The structural and functional scans were normalized to the standard SPM Montreal Neurological Institute template gray prior probability map via the individuals' segmented gray matter image. Functional scans were then smoothed with an 8-mm full-width half-maximum Gaussian kernel.

Seed-to-whole brain connectivity analysis. In these same RA patients, we have previously shown that DMN-left mid/ posterior insula connectivity is associated with the FM survey criteria score (2) and that the left IPL is more connected to mul- tiple brain networks in patients with high levels of inflammation (4). To determine how FM and inflammation interact in the brain, we used these same left insular and IPL clusters as seed regions (Figures $1 \mathrm{~A}$ and $2 \mathrm{~A}$ ). The preprocessed functional data were entered into a Functional Connectivity Toolbox version 15 (CONN; Cognitive and Affective Neuroscience Laboratory, Massachusetts Institute of Technology, Cambridge [http://www.nitrc.org/projects/ conn]). As previously described (2,4), a component-based method (CompCor) was performed to remove confounding effects. The confounds included 6 subject-specific motion parameters, and the signal from white matter and cerebrospinal fluid and their first-order derivatives. A band-pass filter $(0.01-0.1 \mathrm{~Hz})$ was applied to remove linear drifts and high-frequency noise in the data. Task and rest onset periods were modeled and concatenated in first-level analyses to generate beta maps representing connectivity between the insula and IPL and the rest of the brain during task and rest conditions. The resulting beta maps were passed onto second-level group analyses in SPM8. Rest periods were not analyzed at the group level because the summed resting condition (2 minutes) is too short for reliable connectivity estimates (8).

Using multiple regression models, the FM+ and FM- groups were assessed independently in SPM8. In each group we assessed the relationship between seed-to-whole brain connectivity and ESR, with age and sex considered covariates of no interest.
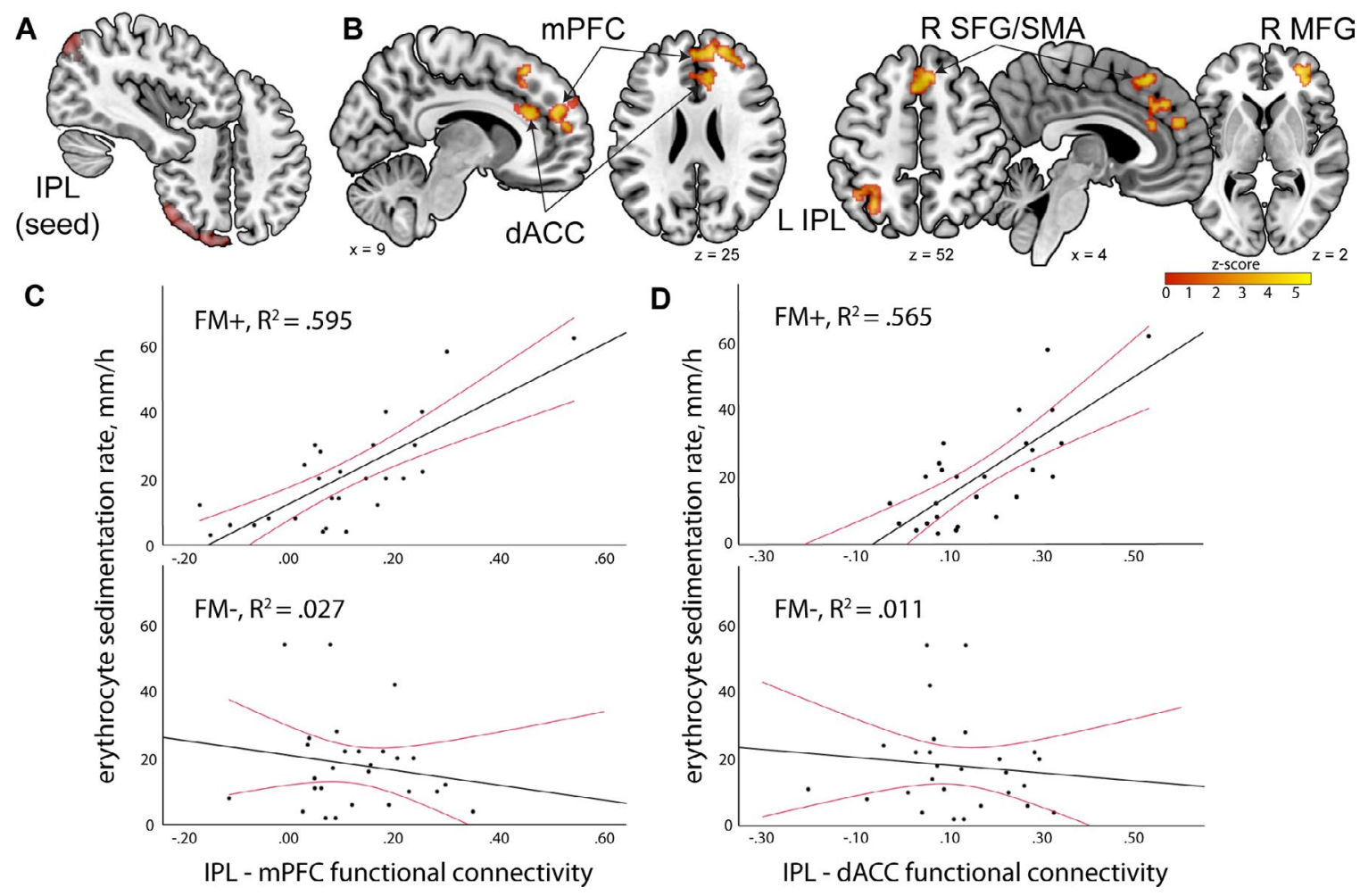

Figure 2. Association of inflammation with functional connectivity in RA patients with concomitant FM. A and $\mathbf{B}$, In RA patients with FM, there was a significant relationship between peripheral inflammation and functional connectivity between the left IPL (A) and the dorsal anterior cingulate cortex (dACC), medial prefrontal cortex (mPFC), left IPL (adjacent to seed region), right superior frontal gyrus/supplementary motor area (R SFG/ $\mathrm{SMA})$, and right MFG (B). C and $\mathbf{D}$, Scatterplots showing the correlation between erythrocyte sedimentation rate and IPL-mPFC connectivity (C) and IPL-dorsal ACC connectivity (D) in RA patients with or without FM; 95\% confidence intervals are displayed in red. See Figure 1 for other definitions. 
The resulting maps were thresholded at an uncorrected voxelwise level of $P<0.001$, and cluster-level family-wise error rates were considered significant at $P<0.05$ after correction for multiple comparisons. The average Fisher-transformed $r$ values of significant clusters were extracted from the first-level beta maps for each patient for secondary analyses to test for interaction effects.

Post hoc interaction analyses. To formally test the possibility of interaction effects, we examined whether the relationship between connectivity values extracted from significant clusters (as described above) and ESR changes as a function of total FM scores. Total scores, rather than FM clinical cutoffs, were used as we have previously shown that this construct can be used continuously (2). ESR and FM survey scores were standardized and centered. These analyses were conducted with general linear models in R version 3.4.4. Post hoc Spearman's rank correlations between functional connectivity and C-reactive protein (CRP) level were calculated to confirm the relationships observed with ESR.

\section{RESULTS}

Clinical characteristics. The clinical characteristics of this cohort have been described previously $(2,4)$ and are shown for FM+ and FM- groups separately (Supplementary Table 1, available on the Arthritis \& Rheumatology web site at http:// onlinelibrary.wiley.com/doi/10.1002/art.41069/abstract). Twenty-seven RA patients met criteria for FM. The mean \pm SD FM score was $17.89 \pm 4.77$ in the FM+ RA group and $8.52 \pm$ 3.19 in the FM- RA group. There was no significant difference in age, sex, or ESR between FM+ and FM- RA patients.
Seed-to-whole brain connectivity analyses. In FM+ RA patients, higher levels of peripheral inflammation were associated with increased functional connectivity between the insula and the left midfrontal gyrus (MFG) and the left IPL (Figures 1B-D and Table 1). Further, in FM+ RA patients, higher peripheral inflammation was associated with increased functional connectivity between the left IPL and multiple cortical regions, including the dorsal anterior cingulate cortex (ACC), medial prefrontal cortex (mPFC), right MFG, right superior frontal gyrus/supplementary motor area, and left IPL (adjacent to the seed IPL region) (Figures 2B-D and Table 1). Of the 7 connections associated with ESR in FM+ patients, 4 were significantly or close to significantly associated with CRP level (Supplementary Table 2, available on the Arthritis \& Rheumatology web site at http://onlinelibrary.wiley.com/doi/10.1002/ art.41069/abstract). In FM- RA patients, higher levels of peripheral inflammation were correlated with lower functional connectivity between the insula and right IPL and higher functional connectivity between the IPL and left superior temporal gyrus (Table 1).

It is possible that other aspects of disease activity, such as the swollen and tender joint counts that are incorporated into the Disease Activity Score in 28 joints (9), could have been affecting our results. We therefore conducted partial correlation analyses between the extracted connectivity values and ESR, while controlling for swollen and tender joint counts, separately in the $\mathrm{FM}+$ and FM- groups. There were no appreciable changes in the strength of the association between ESR and connectivity in these analyses (data not shown). Additionally, adjustment for body mass index did not alter the associations between ESR and connectivity in either group (data not shown).

Table 1. Associations between seed-to-whole brain functional connectivity and erythrocyte sedimentation rate*

\begin{tabular}{|c|c|c|c|c|c|c|c|}
\hline \multirow{2}{*}{$\begin{array}{c}\text { Seed region } \\
\text { cluster (direction of association) }\end{array}$} & \multicolumn{3}{|c|}{ MNI coordinates } & \multirow[b]{2}{*}{ Z score } & \multirow{2}{*}{$\begin{array}{l}\text { Cluster size, } \\
\text { no. of voxels }\end{array}$} & \multirow{2}{*}{$\begin{array}{l}P, \text { FWE- } \\
\text { corrected }\end{array}$} & \multirow{2}{*}{$\begin{array}{l}P \text {, post hoc } \\
\text { interaction }\end{array}$} \\
\hline & $x$ & Y & Z & & & & \\
\hline \multicolumn{8}{|l|}{ With fibromyalgia } \\
\hline \multicolumn{8}{|l|}{ Left insula } \\
\hline Left midfrontal gyrus (+) & -36 & 24 & 32 & 4.38 & 161 & 0.007 & 0.049 \\
\hline Left IPL $(+)$ & -50 & -50 & 40 & 3.98 & 132 & 0.019 & 0.015 \\
\hline \multicolumn{8}{|l|}{ Left IPL } \\
\hline Bilateral dorsal ACC/mPFC (+) & 2 & 38 & 36 & 4.43 & 239 & 0.001 & 0.016 \\
\hline Right midfrontal gyrus (+) & 30 & 48 & 2 & 4.31 & 132 & 0.026 & 0.317 \\
\hline Bilateral SFG/SMA (+) & 8 & 28 & 52 & 4.15 & 128 & 0.030 & 0.040 \\
\hline Right mPFC/right dorsal ACC (+) & 12 & 60 & 30 & 3.88 & 349 & 0.000 & 0.002 \\
\hline Left IPL (+)† & -32 & -48 & 46 & 3.76 & 135 & 0.023 & 0.016 \\
\hline \multicolumn{8}{|l|}{ Without fibromyalgia } \\
\hline \multicolumn{8}{|l|}{ Left insula } \\
\hline Right IPL/precuneus/angular (-) & 28 & -52 & 44 & 4.51 & 175 & 0.002 & $<0.001$ \\
\hline \multicolumn{8}{|l|}{ Left IPL } \\
\hline Left STG (+) & -50 & -42 & 6 & 4.29 & 257 & 0.000 & 0.024 \\
\hline
\end{tabular}

* MNI = Montreal Neurological Institute; FWE = family-wise error; ACC = anterior cingulate cortex; $\mathrm{mPFC}=$ medial prefrontal cortex; SFG = superior frontal gyrus; SMA = supplementary motor area; STG = superior temporal gyrus.

† Adjacent to the seed inferior parietal lobule (IPL) region. 
Post hoc interaction analyses. There was a significant interaction effect between FM scores and ESR for each extracted connectivity value except for the left IPL-right MFG $(P=0.317)$ (Table 1). This suggests that the interaction between FM symptoms and inflammation is important irrespective of whether a patient meets epidemiologic criteria for FM.

\section{DISCUSSION}

In RA, peripheral inflammation appears to affect FM status as reflected on measures of brain functional connectivity. In our previous study (2), we demonstrated that a neurobiologic signature of pain centralization (enhanced insula-DMN connectivity) is also present in RA. Importantly, across the overall sample, this connectivity was not significantly associated with peripheral inflammation. In this study, we show for the first time that inflammation is neurobiologically associated with other more established patterns of pronociceptive connectivity in RA, but this is observed only in patients with more FM symptoms. These effects were apparent when FM was measured as a continuous construct, suggesting that FM symptoms may have important neurobiologic correlates irrespective of whether a patient meets clinical criteria for FM, as we have previously reported (2). This in turn suggests that there is an interaction between peripherally enhanced sensitization and centrally maintained sensitization that contributes to FM symptoms in RA. Put simply, how the brain responds to peripheral inflammation may be critical in understanding why some RA patients have high levels of concomitant FM symptoms while others do not.

The insula is a multimodal sensory processing region and is critically involved in pain perception (10). In FM+ RA patients, increased insula-left IPL connectivity was positively correlated with peripheral inflammation. The IPL is a key node of the DMN, and heightened DMN-insula connectivity is an established neurobiologic feature of FM (10), as well as of these same RA patients with high levels of FM symptoms (2). In FM+ RA patients, we would expect to see increased DMN-insula functional connectivity, but the relationship with peripheral inflammation observed in this study is novel. Interestingly, this left IPL region overlapped with the IPL cluster we previously identified as being more strongly connected to multiple brain networks in patients with high levels of peripheral inflammation (4).

Using the IPL cluster from our previous study (4) as a seed region, we found that IPL-dorsal ACC and IPL-mPFC functional connectivity was positively associated with inflammation only in $\mathrm{FM}+\mathrm{RA}$ patients. It has been previously shown that during the completion of a painful task, FM patients exhibit higher activity in the dorsal ACC compared to healthy participants (11). A previous study in RA patients demonstrated that the dorsal ACC, mPFC, and left IPL were activated during a provoked joint pain paradigm, and that the $\mathrm{MPFC}$ and left IPL regions were functionally connected during this painful task (12). In this context, our results may indicate preliminary evidence of the integration of inflammation-linked brain connectivity and classic pronociceptive brain connectivity.
In FM- patients, insula-right IPL functional connectivity was negatively correlated with peripheral inflammation, while insula-left IPL functional connectivity was positively correlated with peripheral inflammation in FM+ patients. There is evidence of hemispheric asymmetry in IPL function (13) and in the susceptibility to neurodegeneration. For instance, the left hemisphere is affected earlier and more severely in Alzheimer's disease, and the left IPL, compared to the right IPL, shows greater metabolic dysfunction in early dementia (13). The left IPL may be more susceptible to peripherally enhanced sensitization, which is why inflammationassociated connectivity changes in this region are predominantly observed in patients with concomitant FM.

This is the first study to examine patterns of inflammationassociated brain connectivity in the context of concomitant FM in RA. Although the evidence is limited, the coexistence of FM symptoms with another disorder is thought to constitute 2 broad processes: 1) a "bottom-up" form of central sensitization, driven primarily by ongoing nociceptive input and somewhat responsive to treatments that target peripheral nociceptive drivers and 2) a "top-down" central sensitization subtype, which is primarily central in origin and unaffected by manipulations of peripheral nociception (3). Consistent with this hypothesized distinction, a previous osteoarthritis study indicated that successful joint replacement surgery resulted in a substantial improvement of responses to experimental pain measures outside the surgical site and that patients whose surgery eliminated their pain exhibited a more normal inhibitory response to evoked pain than they did prior to surgery (14). In RA, an analogous phenomenon would involve improvement in concomitant FM symptoms following successful inhibition of inflammation. Future studies will focus on patterns of inflammation-associated brain connectivity that predict improvement in concomitant FM after inflammationreducing therapy.

This study has some limitations. First, all of the RA patients in the study had significant levels of chronic fatigue, which may not necessarily be representative of all patients and could have biased the sample toward a centralized pain phenotype. Second, we examined functional connectivity during the performance of a cognitive task from 2 seed regions based on earlier results from the same cohort. Future studies should attempt a whole brain agnostic approach in an independent sample in which patients are at rest or performing other tasks. We do not know when patients developed FM symptoms relative to the onset of RA. Therefore, some patients may belong to a different phenotype wherein "top-down" central sensitization exists prior to RA diagnosis. We have made the reasonable assumption that our measure of systemic inflammation (ESR) is derived entirely from the well-established peripheral immune dysfunction that characterizes RA. However, considering the evolving evidence that supports a neural inflammatory reflex (15), it is also possible that altered brain connectivity drives peripheral inflammation, perhaps via endocrine pathways. It would be intriguing to evaluate 
the responsiveness of identified pronociceptive connectivity in the context of a peripherally directed antiinflammatory treatment, as mentioned above.

In a small study on tumor necrosis factor inhibitors (TNFi) in RA, Rech et al demonstrated that pronociceptive brain connectivity decreased rapidly (i.e., 3 days) after commencement of TNFi treatment and before a positive clinical response was observed (16), providing some support for the concept of "bottom-up" sensitization. Ultimately, the great clinical value of distinguishing "bottom-up" mechanisms of central sensitization is the potential to identify RA patients whose FM symptoms may improve with an enhanced peripheral immunosuppressive approach. In contrast, "top-down" central sensitization would not be expected to respond to peripherally directed therapies. Instead, patients may receive benefit from centrally acting interventions. Until more definitive studies are conducted, these concepts remain as working hypotheses.

Our finding that peripheral inflammation enhances connectivity in established pronociceptive pathways provides preliminary neurobiologic evidence of a distinct FM-like subtype in RA. Identifying the neurobiologic patterns associated with different forms of central sensitization of FM in RA may help to refine therapeutic targets.

\section{ACKNOWLEDGMENTS}

We would like to thank the patients who volunteered to be part of this research effort and Mariella D'Allesandro for help with recruitment and data collection.

\section{AUTHOR CONTRIBUTIONS}

All authors were involved in drafting the article or revising it critically for important intellectual content, and all authors approved the final version to be published. Dr. Kaplan had full access to all of the data in the study and takes responsibility for the integrity of the data and the accuracy of the data analysis.

Study conception and design. Kaplan, Schrepf, Ichesco, Larkin, Harte, Harris, Murray, Waiter, Clauw, Basu.

Acquisition of data. Murray, Waiter, Basu.

Analysis and interpretation of data. Kaplan, Schrepf, Ichesco, Larkin, Harte, Harris, Murray, Waiter, Clauw, Basu.

\section{ROLE OF THE STUDY SPONSOR}

Pfizer had no role in the study design or in the collection, analysis, or interpretation of the data, the writing of the manuscript, or the decision to submit the manuscript for publication. Publication of this article was not contingent upon approval by Pfizer.

\section{REFERENCES}

1. Boyden SD, Hossain IN, Wohlfahrt A, Lee YC. Non-inflammatory causes of pain in patients with rheumatoid arthritis. Curr Rheumatol Rep 2016;18:30.

2. Basu N, Kaplan CM, Ichesco E, Larkin T, Harris RE, Murray A, et al. Neurobiologic features of fibromyalgia are also present among rheumatoid arthritis patients. Arthritis Rheumatol 2018;70:1000-7.

3. Harte SE, Harris RE, Clauw DJ. The neurobiology of central sensitization. J Appl Biobehav Res 2018;23:e12137.

4. Schrepf A, Kaplan CM, Ichesco E, Larkin T, Harte SE, Harris RE, et al. A multi-modal MRI study of the central response to inflammation in rheumatoid arthritis. Nat Commun 2018;9:2243.

5. Aletaha D, Neogi T, Silman AJ, Funovits J, Felson DT, Bingham CO III, et al. 2010 rheumatoid arthritis classification criteria: an American College of Rheumatology/European League Against Rheumatism collaborative initiative. Arthritis Rheum 2010;62:2569-81.

6. Chalder T, Berelowitz G, Pawlikowska T, Watts L, Wessely S, Wright $D$, et al. Development of a fatigue scale. J Psychosom Res 1993;37:147-53.

7. Wolfe F, Clauw DJ, Fitzcharles MA, Goldenberg DL, Häuser W, Katz $\mathrm{RS}$, et al. Fibromyalgia criteria and severity scales for clinical and epidemiological studies: a modification of the ACR Preliminary Diagnostic Criteria for Fibromyalgia. J Rheumatol 2011;38:1113-22.

8. Van Dijk KR, Hedden T, Venkataraman A, Evans KC, Lazar SW, Buckner RL. Intrinsic functional connectivity as a tool for human connectomics: theory, properties, and optimization. J Neurophysiol 2010;103:297-321.

9. Prevoo ML, van 't Hof MA, Kuper HH, van Leeuwen MA, van de Putte LB, van Riel PL. Modified disease activity scores that include twenty-eight-joint counts: development and validation in a prospective longitudinal study of patients with rheumatoid arthritis. Arthritis Rheum 1995;38:44-8.

10. Napadow V, LaCount L, Park K, As-Sanie S, Clauw DJ, Harris RE. Intrinsic brain connectivity in fibromyalgia is associated with chronic pain intensity. Arthritis Rheum 2010;62:2545-55.

11. Pujol J, López-Solà M, Ortiz H, Vilanova JC, Harrison BJ, Yücel M, et al. Mapping brain response to pain in fibromyalgia patients using temporal analysis of FMRI. PLoS One 2009;4:e5224.

12. Schweinhardt P, Kalk N, Wartolowska K, Chessell I, Wordsworth $P$, Tracey I. Investigation into the neural correlates of emotional augmentation of clinical pain. Neuroimage 2008;40:759-66.

13. Toga AW, Thompson PM. Mapping brain asymmetry. Nat Rev Neurosci 2003;4:37-48.

14. Kosek E, Ordeberg G. Lack of pressure pain modulation by heterotopic noxious conditioning stimulation in patients with painful osteoarthritis before, but not following, surgical pain relief. Pain 2000;88:69-78.

15. Pavlov VA, Tracey KJ. Neural regulation of immunity: molecular mechanisms and clinical translation. Nat Neurosci 2017;20:156-66.

16. Rech J, Hess A, Finzel S, Kreitz S, Sergeeva M, Englbrecht M, et al. Association of brain functional magnetic resonance activity with response to tumor necrosis factor inhibition in rheumatoid arthritis. Arthritis Rheum 2013;65:325-33. 